\title{
Traumatic brain injury (TBI) in older adults: aging with a TBI versus incident $\mathrm{TBI}$ in the aged
}

Approximately 39 million older adults (age $\geq 65$ ) were evaluated for traumatic brain injury (TBI) in United States emergency departments during the 2-year period from 2009 to 2010 , representing a $61 \%$ increase in estimates from prior years (Albrecht et al., 2015a). Across the lifespan, an estimated 5.3 million Americans are living with a TBI-related disability (Centers for Disease Control and Prevention (CDC), 2003). With improved recognition and management, more individuals experiencing TBI are surviving to die of other causes later in life (Flanagan et al., 2005). Taken together, these statistics highlight two important populations: those who are "aging with a TBI" and "incident TBI in the aged."

Although the population aging with a TBI is increasingly studied, it is difficult to comment on the role of a remote TBI (e.g. a motor vehicle accident with loss of consciousness at age 20) in an older patient presenting with new onset cognitive or other neuropsychiatric symptoms (NPS) (e.g. depressive symptoms at age 68). In contrast, incident TBI in the aged is understudied, despite the closer temporal relationship between TBI (e.g. fall with loss of consciousness at age 67) and new onset of cognitive or other NPS (e.g. depressive symptoms at age 68). The vast majority of recommendations for diagnosis, prognosis, and management of incident TBI in older adults is generalized from research performed in younger adults. After a brief discussion of aging with TBI, this editorial will focus on older adults with incident TBI and make the point that a "one-size-fits-all" model is inadequate.

\section{Aging with a TBI}

The study of how TBI impacts the aging process is difficult, with most research taking a crosssectional approach given the logistic and monetary implications of multi-decade studies. Evidence suggests that, in a subset of individuals, the cognitive and other NPS that manifest acutely after TBI may persist throughout life (Flanagan et al., 2005). In addition to these sustained changes, there is evidence that some with TBI experience age-related cognitive decline prematurely. One mechanism by which TBI may influence the aging process is microglial priming (Ziebell et al., 2016). This hypothesis posits that microglia, the innate immune cells of the central nervous system, develop and maintain a "pro-inflammatory profile" that is sustained long after the acute effects of TBI have dissipated. This "priming" may set the stage for glial dysfunction and hyperactivity with subsequent challenges. Assuming that the brain has a finite capacity for recovery and adaptation, and that both TBI and aging utilize the same plasticity mechanisms, after a TBI the brain may have fewer resources to devote to the aging process. This should not be thought of as the onset of a new neurodegenerative process, but rather a change in the way the brain handles "normal" aging.

Perhaps the most debated topic in those aging with a TBI is its impact on the development and progression of neurodegenerative processes with some literature showing a firm correlation and others a lack thereof. Data from the National Alzheimer's Coordinating Center (NACC) suggest that TBI may be a risk factor for earlyonset Alzheimer's disease ( $\mathrm{AD}$; onset $<65$ years) (Mendez et al., 2015) and that disinhibition is more likely to occur in those with $\mathrm{AD}$ who have a history of TBI. This association between pre-dementia TBI and disinhibition has been shown previously (Rao et al., 2010). However, in a recent meta-analysis, no statistically significant association was found between TBI and mild cognitive impairment or AD (Crane et al., 2016). An association was found between TBI and the progression of Parkinsonian signs or risk for incident Parkinson's disease. Put simply, it is likely that in a subset of individuals with a predisposition to develop a neurodegenerative disorder, TBI influences the timing and nature of onset and progression. It is also likely that a separate neurodegenerative process exists, the term chronic traumatic encephalopathy (CTE) has been used when discussing repetitive TBI, and that without TBI would not occur in a given individual. An example would be an individual with a genetic predisposition for tau tangle formation that without an inciting event for abnormal tau deposition (e.g. after a TBI) would not develop a tauopathy. Given 
the wide array of mechanisms that may be involved in this process, and the debate in the field over the existence of CTE, this topic warrants an editorial all its own.

\section{Incident TBI in the aged}

There remains a major gap in the study of incident TBI in the aged. This is particularly problematic as the number of TBIs in older adults will continue to increase with the aging of the population. Historically, it was accepted that at all severities of TBI, when compared to younger, older individuals are more likely to be hospitalized, recover more slowly, and exhibit worse outcomes (Staples et al., 2016). However, this is a difficult comparison as there are a number of important demographic and clinical differences between these two groups, and the routinely used outcome measures were developed and validated in younger cohorts. There is likely a subset of older individuals with TBI that have outcomes similar to younger. However, more research is needed to reliably identify these individuals (Mak et al., 2012).

When focused on older adults presenting to the hospital after blunt head trauma, the most common race is white and the sexes present in equal numbers (Flanagan et al., 2005). In younger adults, males are more likely than females to present. Compared to younger individuals, older individuals are more likely to be insured and to have at least one chronic medical condition, including cerebrovascular or cardiorespiratory disease, diabetes, cognitive impairment, musculoskeletal disease, and hearing/visual impairment (Thompson et al., 2006; Staples et al., 2016). Importantly, these conditions may impact the reliability of assessments. As part of routine management for these chronic conditions, older adults are more likely to be on medications, including aspirin and anticoagulants. Chronic conditions and medications can compound injury and complicate surgical management (Thompson et al., 2006). Focusing on psychiatric diagnoses, older adults experiencing TBI are less likely to have a previously diagnosed psychiatric disorder (Deb and Burns, 2007).

Older adults also have distinct injury characteristics. Falls are by far the leading cause of TBI in older adults compared to motor vehicle accidents and assaults in younger adults (Thompson et al., 2006). Given that history of even a single fall is a major risk factor for subsequent fall, it is possible that older adults are at a higher risk of subsequent and repetitive TBI; however, this has not been adequately studied. Older adults are more likely to have complicated TBIs with intracranial bleeding, even with TBIs that would otherwise be classified as mild (Papa et al., 2012). With age, the dura become more adherent to the skull, there is worse cerebrovascular atherosclerosis, and bridging veins are more susceptible to shearing (Thompson et al., 2006). With brain atrophy, whether "normal for age" or in the setting of neurodegeneration, there is increased space in the cranial vault for accumulation of blood, which may result in an intact neurological examination despite occult findings on head computed tomography.

In terms of clinical course and outcome, upon presenting to an emergency department for evaluation of blunt head trauma, older adults are more likely to receive a head computerized tomography (CT) scan. The American College of Emergency Physicians criteria for neuroimaging and decision making in adults presenting with blunt head trauma include older age $(\geq 60$ if loss of consciousness or post-traumatic amnesia, $\geq 65$ if neither) as an indication for CT scan (Jagoda et al., 2009). Older adults are more likely to be hospitalized, and once hospitalized, older adults are more likely to have extended hospitalizations, be transferred to a nursing facility after discharge, and be more severely disabled at the time of discharge than younger adults (Flanagan et al., 2005). Mortality rates for older people with mild TBI are substantially higher than for younger counterparts. Global outcome measures also indicate that older adults have greater dependence after TBI (Thompson et al., 2006). Older adults are more likely to be unemployed postTBI and are more likely to retire because of injury (Ritchie et al., 2014).

Differences in cognitive and other NPS between younger and older adults with incident TBI have received little attention. Although moderate-tosevere TBI with incidence at any age has been consistently associated with increased dementia risk, mild TBI has only been associated with incidence in older adults (i.e. younger adults with mild TBI are not at an increased dementia risk) (Gardner et al., 2014). One study showed that the most prevalent NPS in younger adults experiencing TBI were irritability and sleep problems, whereas in older adults, poor memory, dependence, and slowness of thinking were most prevalent (Deb and Burns, 2007). Looking at Medicare data, TBI significantly increases the risk of new onset depression post-TBI among older adults with evidence of under-recognition and under-treatment (Albrecht et al., 2015b). Older age is also associated with poorer social integration and quality of life post-TBI, although there are co-morbidities more common in older adults that influence this (Deb 
and Burns, 2007). On a more positive note, Mosenthal et al. found older adults with mild TBI to have similar functional outcomes at discharge and 6-month follow-up with the majority of patients able to live independently with measurable improvement in functional status during the first 6 months after injury (Mosenthal et al., 2004). In this same study, older adults with moderate-to-severe TBI did worse than their younger counterparts.

Another point to be made is that measures used to diagnose TBI and evaluate outcomes were developed for younger cohorts making it difficult to know how applicable they are to older adults. By way of example, the Glasgow Coma Score (GCS), a routine part of most diagnostic criteria for TBI, is a reliable predictor of morbidity and mortality in younger but not older adults (Salottolo et al., 2014). Although this may mean that it is harder to predict outcome in older adults, it may also suggest that (1) a modification of the GCS scale would be helpful when applied to older adults or (2) a better scale is needed overall as the GCS is most useful for assessment of outcome in moderate-to-severe TBI, not mild TBI. Just as many of the best-established neurocognitive scales have established norms based on age and other demographics, something similar may be needed when looking at outcomes in TBI.

\section{Conclusion}

New onset TBI in older individuals is a critical area of study that will become more important as the population ages. This group of individuals should be separated from those aging with a TBI. The issue is further complicated by the concepts of brain/biological and cognitive/neural reserve, both of which help to explain the variability of "normal" aging (Mathias and Wheaton, 2015). Brain reserve is a passive model of reserve, where reserve derives from brain size or neuronal count, whereas cognitive reserve suggests that the brain actively attempts to cope with brain damage by using pre-existing cognitive processing approaches or by enlisting compensatory approaches (Bigler and Stern, 2015). The concept of reserve is very interesting: By studying incident TBI in the elderly, we stand to learn more about these processes. In short, a "one-size-fits-all" approach to the conceptualization, diagnosis, and management of TBI across the lifespan is inadequate.

\section{Conflict of interest}

None.

\section{Matthew E. Peters}

Department of Psychiatry and Behavioral Sciences, Johns Hopkins University School of Medicine, Baltimore, MD, USA

\section{References}

Albrecht, J. S. et al. (2015a). Increased rates of mild traumatic brain injury among older adults in US emergency departments, 2009-2010. Fournal of Head Trauma Rehabilitation, 31, E1-E7.

Albrecht, J. S. et al. (2015b). Patterns of depression treatment in medicare beneficiaries with depression after traumatic brain injury. Fournal of Neurotrauma, 32, 1223-1229.

Bigler, E. D. and Stern, Y. (2015). Traumatic brain injury and reserve. Handbook of Clinical Neurology, 128, 691-710.

Centers for Disease Control and Prevention (CDC), N. C. f. I. P. a. C. (2003). Report to congress on mild traumatic brain injury in the United States: steps to prevent a serious public health problem. Atlanta, GA: Centers for Disease Control and Prevention.

Crane, P. K. et al. (2016). Association of traumatic brain injury with late-life neurodegenerative conditions and neuropathologic findings. $\mathcal{F} A M A$ Neurology, 73, 1062-1069.

Deb, S. and Burns, J. (2007). Neuropsychiatric consequences of traumatic brain injury: a comparison between two age groups. Brain Injury, 21, 301-307.

Flanagan, S. R., Hibbard, M. R. and Gordon, W. A. (2005). The impact of age on traumatic brain injury. Physical Medicine and Rehabilitation Clinics of North America, 16, 163-177.

Gardner, R. C., Burke, J. F., Nettiksimmons, J., Kaup, A., Barnes, D. E. and Yaffe, K. (2014). Dementia risk after traumatic brain injury versus nonbrain trauma: the role of age and severity. $\mathcal{F A M A}$ Neurology, 71, 1490-1497.

Jagoda, A. S. et al. (2009). Clinical policy: neuroimaging and decisionmaking in adult mild traumatic brain injury in the acute setting. Fournal of Emergency Nursing, 35, e5-e40.

Mak, C. H. et al. (2012). Traumatic brain injury in the elderly: is it as bad as we think? Current Translational Geriatrics and Experimental Gerontology Reports, 1, 171-178.

Mathias, J. L. and Wheaton, P. (2015). Contribution of brain or biological reserve and cognitive or neural reserve to outcome after TBI: a meta-analysis (prior to 2015). Neuroscience and Biobehavioral Reviews, 55, 573-593.

Mendez, M. F., Paholpak, P., Lin, A., Zhang, J. Y. and Teng, E. (2015). Prevalence of traumatic brain injury in early versus late-onset Alzheimer's disease. Fournal of Alzheimers Disease, 47, 985-993.

Mosenthal, A. C. et al. (2004). The effect of age on functional outcome in mild traumatic brain injury: 6-month report of a prospective multicenter trial. The Fournal of Trauma, 56, 1042-1048.

Papa, L., Mendes, M. E. and Braga, C. F. (2012). Mild traumatic brain injury among the geriatric population. Current Translational Geriatrics and Experimental Gerontology Reports, 1, 135-142. 
Rao, V. et al. (2010). Neuropsychiatric symptoms in dementia patients with and without a history of traumatic brain injury. The fournal of Neuropsychiatry and Clinical Neurosciences, 22, 166-172.

Ritchie, L., Wright-St Clair, V. A., Keogh, J. and Gray, M. (2014). Community integration after traumatic brain injury: a systematic review of the clinical implications of measurement and service provision for older adults. Archives of Physical Medicine and Rehabilitation, 95, 163-174.

Salottolo, K., Levy, A. S., Slone, D. S., Mains, C. W. and Bar-Or, D. (2014). The effect of age on glasgow coma scale score in patients with traumatic brain injury. $\mathcal{F} A M A$ Surgery, 149, 727-734.
Staples, J. A., Wang, J., Zaros, M. C., Jurkovich, G. J. and Rivara, F. P. (2016). The application of IMPACT prognostic models to elderly adults with traumatic brain injury: a population-based observational cohort study. Brain Injury, 30, 899-907.

Thompson, H. J., McCormick, W. C. and Kagan, S. H. (2006). Traumatic brain injury in older adults: epidemiology, outcomes, and future implications. Fournal of the American Geriatrics Society, 54, 1590-1595.

Ziebell, J. M. et al. (2016). Aging with a traumatic brain injury: could behavioral morbidities and endocrine symptoms be influenced by microglial priming? Brain Behavior, and Immunity, 16, 30053-30058. 